\title{
Relevamiento de nematodes gastrointestinales en bovinos del sur de Misiones y nordeste de Corrientes (Argentina)
}

\author{
Lobayan, S.I. ${ }^{1}$; Tuzinkievicz, T.M. ${ }^{1}$; Fiel, C.A. ${ }^{2}$; Nápoli, J.N. ${ }^{3}$; Dalzotto, A.P. ${ }^{3}$ \\ ${ }^{1}$ Escuela de Veterinaria, Sede Virasoro, Univ.Salvador (USAL), Ruta Nac.14, Km 728, Gdor. Virasoro, \\ Corrientes (Argentina). ${ }^{2}$ Univ.Nac.Centro Prov.Bs.As. (UNCPBA). ${ }^{3}$ Alumnos practicantes USAL. \\ E-mail: uds-vira@salvador.edu.ar
}

\begin{abstract}
Resumen
Lobayan, S.I.; Tuzinkievicz, T.M.; Fiel, C.A.; Nápoli, J.N.; Dalzotto, A.P.: Relevamiento de nematodes gastrointestinales en bovinos del sur de Misiones y nordeste de Corrientes (Argentina). Rev. vet. 27: 2, 137-140, 2016. Se realizó un estudio de investigación observacional de nematodes gastrointestinales de bovinos en 592 terneros destete de 12 rodeos de cría durante 27 meses. El área de estudio comprendió los departamentos Apóstoles y Capital (Provincia de Misiones) y departamentos Ituzaingó y Santo Tomé (Provincia de Corrientes), de características fitogeográficas similares. Las variables bajo estudio fueron la cantidad de huevos excretados por medio del método de conteo de huevos por gramo de materia fecal (hpg) y la proporción de los géneros parasitarios detectada mediante coprocultivo e identificación de larvas de tercer estadio. Debido a la distribución asimétrica de la variable, para la valoración del conteo de huevos se utilizó la mediana como parámetro de tendencia central. En el total de las muestras se registró una mediana de $90 \mathrm{hpg}$, y en el análisis por estación se verificaron medianas de $200 \mathrm{hpg}$ en primavera, $190 \mathrm{hpg}$ en verano y $50 \mathrm{hpg}$ en otoño e invierno. El coprocultivo se realizó sobre 21 pools de muestras en 8 estaciones seguidas durante todo el estudio y los hallazgos totales fueron: $30 \%$ Haemonchus sp., $26 \%$ Trichostrongylus $s p ., 24 \%$ Cooperia $s p$. y $20 \%$ Oesophagostomum $s p$. La participación mayoritaria de nematodes de alta oviposición y los relativamente bajos conteos de hpg en heces -exceptuando algunos casos con altos valores- indican la necesidad de profundizar los estudios epidemiológicos de las parasitosis de la región para establecer medidas de control que reduzcan su impacto en las explotaciones ganaderas.
\end{abstract}

Palabras clave: bovino, nematodes gastrointestinales, relevamiento, nordeste argentino.

\begin{abstract}
Lobayan, S.I.; Tuzinkievicz, T.M.; Fiel, C.A.; Nápoli, J.N.; Dalzotto, A.P.: Identification of gastrointestinal nematodes in cattle from Misiones and Corrientes (Argentina). Rev. vet. 27: 2, 137-140, 2016. An observational research of gastrointestinal nematodes of cattle was conducted in 592 weaned calves from 12 breeding herds during 27 months. The area of study comprised the Apóstoles and Capital districts (Misiones Province) and the Ituzaingó and Santo Tomé districts (Corrientes Province), of similar phytogeographic characteristics. Two variables were measured: the number of excreted eggs by the counting method of eggs per gram (epg) of stool, and the proportion of the parasitic genus by stool culture and identification of third stage larvae. In egg counts the median was used because of the asymmetric distribution of the variable as a measure of central tendency, being observed in the total sample a median of $90 \mathrm{epg}$, and in the seasonal analysis a median of $200 \mathrm{epg}$ in spring, 190 epg in summer and 50 epg in autumn and winter. In the stool culture 21 pools of samples were grown in 8 seasons in a row throughout the whole study and the overall findings were: $30 \%$ Haemonchus sp., 26\% Trichostrongylus sp., 24\% Cooperia sp., and 20\% Oesophagostomum $\mathrm{sp}$. The majority participation of high oviposition nematodes, and the relatively low epg counts in faeces -excepting some cases with high values- indicate the necessity to increase the epidemic studies of the regional parasitosis and to establish control measures that reduce their impact in the cattle exploitations.
\end{abstract}

Key words: cattle, gastrointestinal nematodes, survey, Argentinean northeast. 


\section{INTRODUCCIÓN}

En una amplia región que abarca el sur de Brasil, Uruguay y Argentina, las condiciones ambientales son favorables para el desarrollo de nematodes gastrointestinales durante todo el año. Dado que los sistemas de producción se establecen sobre pasturas permanentes, las parasitosis internas representan una de las mayores limitantes ${ }^{14}$.

Las parasitosis gastrointestinales ejercen su efecto por mortandad, enfermedad clínica y/o subclínica. En la pampa húmeda argentina se estiman pérdidas anuales por mortandad del orden de 25.000 a 30.000 tn de carne y pérdidas en la producción de carne del orden de $226.000 \mathrm{tn}$, sumando una pérdida global de unas $260.000 \mathrm{tn}^{7}$.

Los estudios de prevalencia resultan esenciales para determinar la estacionalidad y peligrosidad del problema, posibilitando el posterior desarrollo de alternativas tendientes a evitar el efecto nocivo de los parásitos. En Argentina, investigaciones de este tipo se realizaron en la pampa húmeda ${ }^{7}$ y subhúmeda ${ }^{9}$, así como en la región central ${ }^{5}$ y nordeste ${ }^{1,3}$. Sin embargo, se carece de información para la zona sur de la Provincia de Misiones y nordeste de la Provincia de Corrientes, que comprenden los departamentos Apóstoles y Capital (Misiones) y Santo Tomé e Ituzaingó (Corrientes).

El objetivo del presente trabajo fue obtener información sobre la dinámica poblacional de los géneros de nematodes gastrointestinales de los bovinos en el sur de Misiones y nordeste de Corrientes, por medio de coprología cuantitativa, coprocultivo e identificación de larvas de tercer estadio.

\section{MATERIAL Y MÉTODOS}

Se realizó un estudio de investigación observacional transversal; la unidad muestral fue el ternero destete. Se seleccionaron los rodeos mediante muestreos de conveniencia, y los terneros de cada rodeo por un muestreo simple al azar. El tamaño de la muestra para los rodeos pequeños fue de 10 terneros y de 97 en el más grande. El estudio se realizó desde abril de 2013 hasta junio de 2015, abarcando 8 estaciones.

Se efectuaron 18 muestreos en 12 rodeos de cría y se extrajeron muestras de 592 terneros. El estudio se realizó en 7 rodeos de los departamentos Apóstoles y Capital (Misiones) y 5 rodeos de Santo Tomé e Ituzaingó (Corrientes). La zona del estudio se extiende por el sur de Misiones y el nordeste de Corrientes ${ }^{2}$.

Como primer criterio de inclusión se estableció la utilización de terneros entre 6 y 10 meses de edad. Como segundo criterio de inclusión se estableció que dichos animales no hubieran recibido tratamiento antiparasitario 60 días antes de la prueba.

Se midieron dos variables cuantitativas discretas, el número de huevos por gramo de materia fecal ("hpg") y la proporción de los géneros parasitarios. Para evaluar el número de huevos se utilizó el tradicional recuento en heces por técnica de MacMaster modificada ${ }^{11}$. Los resultados de hpg fueron analizados estadísticamente con el software Infostat versión $2008^{6}$.

Se obtuvieron medidas de resumen sobre el total de las muestras y por años y estaciones, debido a la natural fluctuación biológica de la producción de huevos por los nematodes gastrointestinales ${ }^{13} \mathrm{y}$ a la distribución asimétrica de la variable. Como medida de tendencia central se utilizó la mediana. Se procedió al análisis de la variancia no paramétrica de los resultados por estación de los tres años mediante la prueba de KruskalWallis con un nivel $\alpha$ de 0,05 para encontrar diferencias por estación.

Se obtuvieron tablas de frecuencias de hpg sobre el total y por estaciones de los tres años. Para evaluar la proporción de géneros parasitarios se realizó el coprocultivo de pools de muestras mediante el método de Corticelli \& Lai ${ }^{4}$. Se cultivaron 21 pools de muestras en 8 estaciones seguidas en los 27 meses del estudio.

Se identificaron $100 \mathrm{~L}_{3}$ por cultivo de tricostrongilídeos y se estableció el porcentaje de cada género hallado utilizando las claves de $\mathrm{Niec}^{10}$. Los resultados de la identificación de géneros de tricostrongilídeos se analizaron estadísticamente y se obtuvieron medidas de resumen y tablas de frecuencias de las estaciones de los tres años.

\section{RESULTADOS}

Sobre el total de las 592 muestras, la mediana fue de 90 hpg, con valores mínimos de 0 , máximo de 8260 y desvío estándar de 677. En la Tabla 1 se consignan las medidas de resumen de hpg por años y estaciones.

Tabla 1. Huevos de nematodes por gramo según años y estaciones.

\begin{tabular}{llccccc}
\hline año & estación & $\mathrm{n}$ & mediana & $\mathrm{DE}$ & mín & máx \\
\hline 2013 & otoño & 20 & 105 & 105 & 10 & 370 \\
2013 & invierno & 60 & 55 & 176 & 0 & 1140 \\
2013 & primavera & 10 & 290 & 315 & 40 & 960 \\
2014 & verano & 50 & 190 & 342 & 20 & 2090 \\
2014 & otoño & 78 & 20 & 249 & 0 & 1970 \\
2014 & invierno & 136 & 50 & 160 & 0 & 980 \\
2014 & primavera & 76 & 200 & 961 & 0 & 8260 \\
2015 & otoño & 162 & 100 & 1000 & 0 & 7030 \\
\hline
\end{tabular}

n: cantidad de muestras, DE: desvío estándar, mín: mínimo, máx: máximo.

Tabla 2. Huevos de nematodes por g según estación (en 3 años).

\begin{tabular}{lccccc}
\hline estación & $\mathrm{n}$ & mediana & $\mathrm{DE}$ & mín & máx \\
\hline invierno & 196 & 50 & 164 & 0 & 1140 \\
otoño & 260 & 50 & 834 & 0 & 7030 \\
primavera & 86 & 200 & 909 & 0 & 8260 \\
verano & 50 & 190 & 342 & 20 & 2090 \\
\hline
\end{tabular}

n: cantidad muestras, DE: desvío estándar, mín: mínimo, máx: máximo. 
Tabla 3. Participación (\%) de los géneros de parásitos en los 3 años.

\begin{tabular}{lcccc}
\hline estación & Haemonchus & Cooperia & Trichostrongylus & Oesophagostomum \\
\hline invierno & 31 & 30 & 19 & 20 \\
primavera & 25 & 17 & 36 & 22 \\
verano & 27 & 22 & 32 & 19 \\
otoño & 35 & 28 & 16 & 21 \\
\hline
\end{tabular}

La prueba no paramétrica de Kruskall Wallis estableció que existieron diferencias de hpg por estaciones en los tres años, con alta significancia $(p<0,0001)$, según se detalla en la Tabla 2 .

En los recuentos de huevos, sobre el total de las muestras de los tres años, el $70 \%$ se ubicó entre 0 y $206 \mathrm{hpg}$ y el $13 \%$ entre 207 y $413 \mathrm{hpg}$, con un $14 \%$ de animales sin eliminación de huevos.

Del análisis de las estaciones de los tres años, surge que en otoño el $94 \%$ de los conteos se ubicaron entre 0 y $328 \mathrm{hpg}$, con un $10 \%$ de animales sin eliminación. En invierno el 76\% estuvo entre 0 y $140 \mathrm{hpg}$, con un $16 \%$ de animales que no eliminaron huevos por materia fecal. En primavera el $98 \%$ de los conteos se situó entre 0 y $1376 \mathrm{hpg}$, con el $2 \%$ de animales con conteos negativos. En el verano el $86 \%$ de los conteos se ubicó entre 20 y $434 \mathrm{hpg}$.

En los coprocultivos realizados a lo largo de los 3 años de estudio se registró la participación porcentual de los siguientes géneros: $30 \%$ de Haemonchus sp., $26 \%$ de Trichostrongylus sp., 24\% de Cooperia sp. y $20 \%$ de Oesophagostomum $s p$. En la Tabla 3 se muestra la participación de cada género en cada una de las estaciones de los tres años.

\section{DISCUSIÓN}

La excreción estacional de huevos, predominante en primavera y verano -con algunos valores muy altos en otoño- se explica por la fluctuación biológica de la oviposición ${ }^{13}$. Además, se ha señalado que las hembras de Haemonchus sp. y Oesophagostomum sp. pueden producir entre 5.000 y 10.000 huevos por día, mientras que las de Trichostrongylus $s p$. generan entre 100 y 200 huevos y Cooperia sp. entre 1.000 y 3.000 huevos, por lo cual los trabajos basados exclusivamente en coprología pueden tener algún sesgo debido al diferente potencial biótico de los géneros involucrados, en comparación con los estudios basados en necropsias ${ }^{8}$.

En la evaluación de la proporción de géneros parasitarios, los resultados coinciden con los obtenidos en otras investigaciones realizadas en zonas subtropicales, donde se registró predominio de los géneros Haemonchus sp., Cooperia sp., Trichostrongylus sp. y Oesophagostomum sp., los cuales tienden a desarrollarse y sobrevivir mejor en climas cálidos, lo cual explica su distribución abundante en el nordeste de Argentina, su estacionalidad en áreas templadas y su inexistencia en las áreas patagónicas ${ }^{12}$.

En conclusión, los relativamente bajos conteos de huevos en materia fecal registrados en la mayor par- te de los animales, con solo algunos individuos con cómputos altos, así como la mayoritaria participación de los géneros de alta oviposición ${ }^{8}$, que asumen importancia por su efecto (mortandad, enfermedad clínica y/o subclínica), evidencian la necesidad de profundizar los estudios epidemiológicos sobre las parasitosis de la región para establecer medidas de control que reduzcan su impacto en la ganadería bovina.

Agradecimientos. Por su colaboración en el proyecto al médico veterinario M.A. Da Luz, investigador del INTA Cerro Azul (Misiones, Argentina).

\section{REFERENCIAS}

1. Bonazzi E, Ivancovich J, Eddi C, Gómez B, Luciani C, Toledo H, Lukovich R, Niec R. 1980. Parasitismo gastrointestinal de los bovinos en el centro-este de la Provincia de Formosa (Argentina). Rev Inv Agrop INTA 15: 683696.

2. Cabrera AL. 1971. Fitogeografía de la República Argentina. Boletín Sociedad Argentina Botánica 15: 1-42.

3. Cardona LG, Zurbriggen M, Ramirez L, Laphitz L, Homse A. 1993. Parasitismo gastrointestinal de bovinos en crecimiento en la zona de malezal. Su variación estacional. INTA (www.inta.gob.ar/mercedes) Informe Plan de Trabajo 27: 2651, 1-7.

4. Corticelli B, Lai M. 1963. Studies on the technique of culture of infective larvae of gastrointestinal strongyles of cattle. Acta Med Vet Napoli 9: 347-357.

5. Descarga CO, Kloster AM, Davies P, Marinelli R. 1994. Parasitismo gastrointestinal en novillos en el sudoeste de la Provincia de Córdoba. Rev Arg Prod Anim 14: 227-236.

6. Di Rienzo JA, Casanoves F, Balzarini MG, González L, Tablada M, Robledo CW. 2008. InfoStat, versión 2008. Grupo InfoStat, FCA, Universidad Nacional de Córdoba, Argentina.

7. Entrocasso C. 1988. Epidemiology and control of bovine ostertagiasis in South America. Vet Parasitol 27: 59-65.

8. Hansen J, Perry B. 1994. The epidemiology, diagnosis and control of helminthes parasites of ruminants, Publ. ILRAD (International Laboratory for Research on Animal Diseases), Nairobi, Kenya, 121 p.

9. Lamberti R, Gino L, Calvo C, Pombar A, Benito A, Ferran A. 2000. Análisis de casuística de muestras de materia fecal recibidas en 1996-1999. UNLP, Anuario 2000. http://www.biblioteca.unlpam.edu.ar/pubpdf/anuavet/n2000a04lamberti.pdf

10. Niec R. 1968. Cultivo e identificación de larvas infectantes de nematodes gastrointestinales del bovino y ovino. Manual Técnico $N^{\circ}$, INTA-Argentina, $37 \mathrm{p}$.

11. Roberts FH, O'Sullivan PJ. 1949. Methods for egg counts and larvae cultures for strongyles infesting the gastro-intestinal tract of cattle. Aust J Agr Res 1: 99-102. 
12. Romero JR, Boero CA. 2001. Epidemiología de la gastroenteritis verminosa de los ovinos en las regiones templadas y cálidas de Argentina. Analecta Vet 21: 21-37.

13. Scheuerle M. 2009. Anthelmintic resistance of Haemonchus contortus and the famacha C-method as a tool to delay the development of anthelmintic resistance. http://edoc. ub.uni muenchen.de/10577/1/Scheuerle_Miriam_C.pdf. Ludwig-Maximi-lians Universität (München).
14. Suarez VH, Cristel SL. 2007. Anthelmintic resistance in cattle nematode in the western Pampeana Region of Argentina. Vet Parasit 144: 111-117.

\section{Asociación Cooperadora de la Facultad de Ciencias Veterinarias Universidad Nacional del Nordeste}

Personería Jurídica No 647/92 y 912/00

Sargento Cabral 2139

3400 Corrientes

Argentina

La Asociación Cooperadora de la Facultad de Ciencias Veterinarias de la UNNE fue constituida el 10 de diciembre del año 1991 como entidad de bien público, con el objeto de promover y coadyuvar las actividades científicas, educativas y culturales relacionadas con las Ciencias Veterinarias. En tal sentido, implementa acciones para colaborar con la enseñanza, extensión, actualización y difusión científica que realiza dicha casa de estudios.

\section{Beneficios que brinda a sus asociados:}

- Fotocopias con descuentos especiales en la Fotocopiadora Copias.com que funciona dentro del predio de la Facultad de Ciencias Veterinarias.

- Descuentos para la adquisición de libros de distintas editoriales.

- Descuentos especiales en otros rubros.

Tel. (0379) 4425753 interno 186 • Página Web www.vet.unne.edu.ar•E-mail: cooperadora@vet.unne.edu.ar 